\title{
Disordered structures in lead-free piezoelectrics
}

Patrick Kin Man Tung ${ }^{1}$, Marton Major ${ }^{2}$, Jessica Hudspeth ${ }^{3}$, Christina Hoffmann ${ }^{4}$, John Daniels ${ }^{5}$

${ }^{1}$ School Of Materials Science And Engineering, University Of New South Wales, Sydney, Australia, ${ }^{2}$ Institute of Materials Science, Technical University of Darmstadt, Darmstadt, Germany, ${ }^{3}$ Beamline ID15, European Synchrotron Radiation Facility, Grenoble, France, ${ }^{4}$ TOPAZ beamline, Spallation Neutron Source, Knoxville, United States, ${ }^{5}$ School Of Materials Science And Engineering, University Of New South Wales, Kensington, Australia

E-mail: patrick.tung@unsw.edu.au

Piezoelectric materials are a vital part of technological advancements and are found in a plethora of existing applications. The prevalent material used for such purposes is Lead Zirconate Titanate (PZT), however, global legislations will force the removal of toxic elements, such as lead, in all electronic components [1]. One potential alternative that shows promising properties is the perovskite solid-solution of Sodium Bismuth Titanate, doped with Barium Titanate (NBT-x\%BT). In particular, the composition of NBT-7\%BT shows enhanced piezoelectric properties, where the material sits on a phase boundary between a ferroelectric rhombohedral and a ferroelectric tetragonal phase, known as a morphotropic phase boundary [2]. However, elucidating the origins of the electro-mechanical mechanisms of such materials is not straightforward, as it has been shown there are numerous disordered structures that contribute to the functional properties [3]. Hence, diffuse scattering was employed to directly probe the short- and medium-range structures of NBT-x\%BT.

Single crystal compositions of NBT-0\%BT, NBT-3.6\%BT, NBT-6.9\%BT and NBT-13.1\%BT were chosen to resolve the local structures across the morphotropic phase boundary. Diffuse X-ray scattering experiments were performed at the ID15B beamline of the European Synchrotron Radiation Facility and diffuse neutron scattering experiments were performed at the TOPAZ beamline of the Spallation Neutron Source. 3D diffuse scattering volumes were collected from both experiments as a function of applied in-situ electric field, temperature variation and doping variation.

Results showed 4 prominent scattering features that are apparent in the 3D diffuse scattering volumes, each providing information on a unique small- to medium-range structure.

1. Broad diffuse scattering around Bragg reflection coordinates indicate an atomic size effect from a pseudo-random occupation of sodium and bismuth in the A-site of the perovskite structure.

2. Reflections occurring on the half-integer scattering planes indicate a doubling of the perovskite unit cell through oxygen octahedral rotations in all directions, that result in an average rhombohedral symmetry.

3. Diffuse streaks running along directions that connect the half-integer reflections indicate planar defects. Here, they are most likely 2-dimensional plates of tetragonal symmetry that are 3-4 unit cells thick, permeating a rhombohedral matrix.

4. Satellite reflections that are offset from Bragg reflection coordinates indicate a structural modulation of approximately 40 $\AA$ occurring in the material. Evidence from in-situ electric fields suggest the modulations are due to a torsional strain of the octahedras around the axes.

Furthermore, this presentation will provide an overview of the disordered structural models giving rise to the experimental diffuse scattering signals and how these may affect the macroscopic functional properties of the material.

[1] Rodel, J. et al. (2009). J. Am. Ceram. Soc. 92, 1153-1177.

[2] Daniels, J. (2009). Appl. Phys. Lett. 95, 032904.

[3] Welberry, T. R. (2004) Diffuse x-ray scattering and models of disorder. Oxford Science Publication.

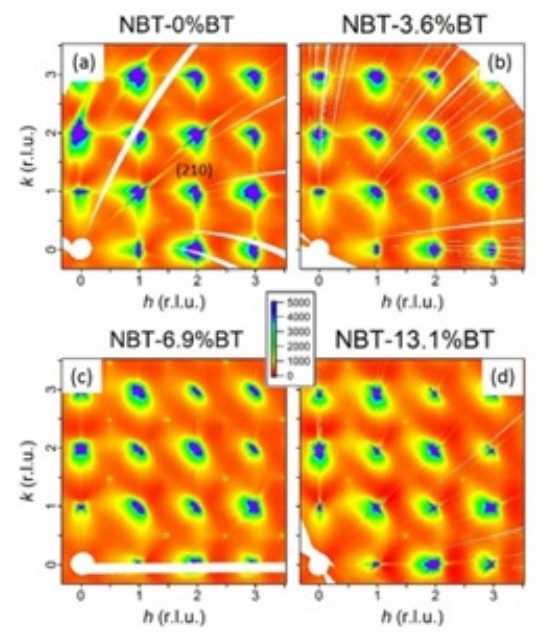

Keywords: lead-free piezoelectrics, diffuse scattering, sodium bismuth titanate 